EPJ Web of Conferences 71, 00082 (2014)

DOI: $10.1051 /$ epjconf / 20147100082

(C) Owned by the authors, published by EDP Sciences, 2014

\title{
Measurements of the production of P-wave charmonium states through radiative decays at the ATLAS experiment
}

\author{
Michal Marcisovsky ${ }^{1, a}$ on behalf of the ATLAS Collaboration \\ ${ }^{1}$ Institute of Physics ASCR Prague
}

\begin{abstract}
The production cross sections of the $\chi_{c 1}$ and $\chi_{c 2}$ charmonium states are measured in $p p$ collisions at $\sqrt{s}=7 \mathrm{TeV}$ using $4.5 \mathrm{fb}^{-1}$ of integrated luminosity recorded by the ATLAS detector at the LHC. The $\chi_{c}$ states are reconstructed via the radiative decay $\chi_{c} \rightarrow J / \psi\left(\mu^{+} \mu^{-}\right) \gamma$, where the photons are reconstructed using $\gamma \rightarrow e^{+} e^{-}$conversion in the detector material. Differential production cross sections for prompt and non-prompt $\chi_{c 1,2}$ are presented, as well as the fraction of inclusive $J / \psi$ produced by feed-down from the $\chi_{c}$ states. The results are compared with a range of theoretical predictions. The branching fraction of $\mathcal{B}\left(B^{+} \rightarrow \chi_{c 1} K^{+}\right)$is measured using the same dataset and $\chi_{c}$ event selection.
\end{abstract}

\section{Introduction}

Quarkonium is a flavorless mesonic bound state of a quark and its anti-quark. The charmonium $(c \bar{c})$ and bottomonium $(b \bar{b})$ systems have been studied particularly deeply both experimentally and theoretically since the discoveries of the $J / \psi$ and $\Upsilon$ mesons in the 1970 s, and a variety of excited states has been found and investigated. Charmonium has a rich energy level spectrum reminiscent of positronium. The $J / \psi$ is perhaps the most widely known state of charmonium, a ${ }^{3} \mathrm{~S}_{1}$ state of the bound $c \bar{c}$ quarks. Due to its relatively large branching ratio of decay into a lepton pair, it is a valuable tool not only for heavy quark studies, but is also a "standard candle" for understanding the detector performance due to the clean experimental signature.

The production of the $c \bar{c}$ pair is well understood in terms of Quantum Chromodynamics (QCD), since heavy quark masses are larger than QCD hadronization scale $\Lambda_{\mathrm{QCD}}$ and perturbative calculations can be applied. On the other hand, the dynamics of the bound state are non-perturbative and it is a natural laboratory for precise tests of effective theories and lattice QCD, since it probes the interplay between the perturbative and non-perturbative regions of QCD - namely the evolution of the $c \bar{c}$ pair into a colourless bound state.

Many other aspects of quarkonium production remain a challenging problem for QCD [1]. The large mass of $c$ and $b$ quarks makes the non-relativistic model description possible. The effective theories - the Color Singlet (CS) model and the Color Octet (CO) model which emerged in the nonrelativistic QCD (NRQCD) were able to explain the transverse momentum $\left(p_{T}\right)$ spectra of the ${ }^{3} S_{1}$ charmonium states observed at the Tevatron and the LHC. However, the polarization predictions of those models disagree with measurements [2]. Currently, no model is able to describe both production cross section and polarization, and therefore the experimental hadron spectroscopy has an essential

a e-mail: marcisov@fzu.cz 
role in understanding of QCD in the quarkonia regimes. The ATLAS detector at the LHC allows the study of such bound states in wide transverse momenta and rapidity regions.

There is only one triplet of the P-wave charmonium states below the open charm threshold, the $\chi_{c J}(1 \mathrm{P})$ with $J^{P C}=0^{++}, 1^{++}, 2^{++}$. The large radiative branching fractions $\mathcal{B}\left(\chi_{c J} \rightarrow J / \psi \gamma\right)=(1.1 \%$, $19.5 \%, 34.4 \%$ ) for $\mathrm{J}=0,1,2$ states make the reconstruction possible [3].

Study of the $\chi_{c}$ production is important, as it represents a significant feed-down fraction in radiative decays of $\chi_{c J} \rightarrow J / \psi \gamma$. Furthermore, the relative production ratio such as $\sigma\left(\chi_{c 2}\right) / \sigma\left(\chi_{c 1}\right)$ is a sensitive measurement for disentangling the $\mathrm{CO}$ contributions to the prompt charmonium production mechanisms [4].

\section{The ATLAS detector}

ATLAS is a general purpose detector at the LHC optimized for high $p_{\mathrm{T}}$ physics and instantaneous luminosity of $10^{34} \mathrm{~cm}^{-2} \mathrm{~s}^{-1}$ with almost $4 \pi$ coverage [5]. The ATLAS detector consists of an Inner Detector (ID) tracker, electromagnetic and hadronic calorimetry systems and a Muon Spectrometer (MS). The key sub-detectors for the study presented in this paper are the ID and the MS. The ID surrounds the interaction point and provides precise information for the reconstruction of charged particle tracks. It consists of a Pixel Detector, a semiconductor microstrip tracker (SCT) and a transition radiation tracker (TRT). For measurements of track momenta and particle charge, the ID is located in a $2 \mathrm{~T}$ magnetic field generated by a superconducting solenoid and covers the pseudorapidity region of $|\eta|<2.5^{1}$. The ID system is enclosed by the liquid-argon lead electromagnetic and the iron scintillator hadronic sampling calorimeters. The MS pseudorapidity coverage extends up to $|\eta|<2.7$, covering a large phase space of the muon transverse momenta up to $1 \mathrm{TeV}$. Muons are reconstructed using the information provided by the MS and the ID.

\section{Measurement of the $\chi_{c J}$ production}

The measurement of the $\chi_{c 1,2}$ differential production cross section is performed using the radiative decay channel $\chi_{c J} \rightarrow J / \psi \gamma$. The measurement is performed in 5 bins of transverse momentum $p_{\mathrm{T}}$ and one bin of rapidity $\left|y_{J / \psi}\right|<0.75$. The $\chi_{c 0}$ cross section is not measured due to its small branching fraction to $J / \psi$ and $\gamma$ and difficult background situation. The measurements presented in this paper are performed using the $4.5 \mathrm{fb}^{-1}$ collected by the ATLAS detector during the $2011 \mathrm{LHC}$ run at a centre-of-mass energy $\sqrt{s}=7 \mathrm{TeV}$. A full description of the measurements can be found in [6].

\subsection{Event selection}

Collision events for this analysis are selected as follows. Two $J / \psi$ candidate muons are required to have both ID and MS track segments and must be of opposite charges. In addition, they must pass the di-muon trigger with a $p_{\mathrm{T}}$ threshold of $4 \mathrm{GeV}$. The ID tracks must satisfy quality requirements by having been reconstructed from at least one Pixel hit and at least six SCT hits, and are required to have originated from a primary vertex built from at least three tracks. The di-muon system invariant mass for the $J / \psi$ candidate must fall within the $2.95<m\left(\mu^{-} \mu^{+}\right)<3.25 \mathrm{GeV}$ window. In addition, the di-muon system must be within the central part of the ATLAS detector with rapidity $\left|y_{\left(\mu^{+} \mu^{-}\right)}\right|<0.75$, since this central region of the detector has the smallest di-muon invariant mass resolution.

\footnotetext{
${ }^{1}$ ATLAS uses a right-handed coordinate system with its origin at the centre of the detector and the z-axis along the beam pipe. The pseudorapidity is defined as $\eta=-\ln \left[\tan \left(\frac{\theta}{2}\right)\right]$, where $\theta$ is the angle between the particle momentum and the beam axis.
} 
For the converted photons, two oppositely charged tracks are required with at least six hits in the SCT subdetector and with $p_{\mathrm{T}}$ at least $400 \mathrm{MeV}$. In addition, the ID tracks must be reconstructed within $|\eta|<2.3$. Track pairs consistent with the photon conversion hypothesis are fitted to a common vertex, which is required to converge with a $\chi^{2} / N_{d o f}<5$. Reconstructed converted photon candidates must have $p_{\mathrm{T}}^{\gamma}>1.5 \mathrm{GeV}$ and must fall within the pseudorapidity range of $\left|\eta^{\gamma}\right|<2$. In addition, the conversion point must be within the Pixel Detector radius of $40<\mathrm{r}<150 \mathrm{~mm}$ to reject fake conversions from $\pi^{0} \rightarrow e^{+} e^{-} \gamma$. An additional requirement on the 3-dimensional impact parameter $a_{0}$ $<5 \mathrm{~mm}$ is imposed on the $J / \psi$ and $\gamma$ candidates to ensure that they come from a common vertex.
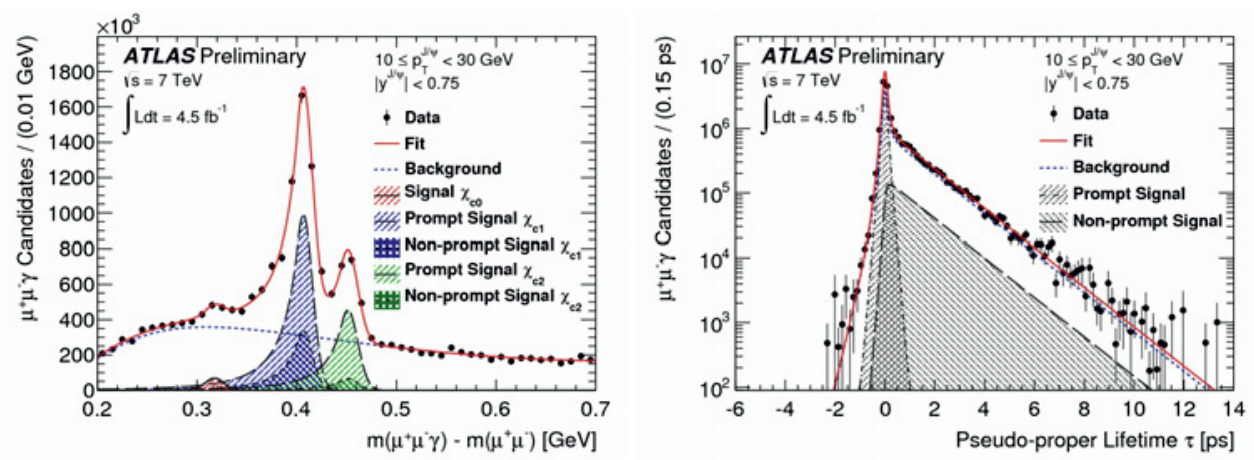

Figure 1. (left) The invariant mass difference $\Delta \mathrm{m}$ and the pseudo-proper lifetime $\tau$ distribution (right) for the $\chi_{c}$ candidates reconstructed within the kinematic region of $10 \leq p_{\mathrm{T}}^{J / \psi}<30 \mathrm{GeV}$ and $\left|y^{J / \psi}\right|<0.75$. A fit to the distribution is indicated by the solid red line. The fitted $\chi_{c 1,2}$ signals are presented as blue and green shaded regions, respectively, on the left plot. The fitted background distributions are shown as the dashed blue lines in both plots [6].

\subsection{The differential $\chi_{c 1}$ and $\chi_{c 2}$ cross section measurements}

The P-wave charmonia are produced directly at the $p p$ collision, emerge in a feed-down from the higher-energy charmonium states such as $\Psi(2 S)$ and also result from decay of b-hadrons. The pseudoproper lifetime $\tau$ is the discriminating factor for the $\chi_{c J}$ production classes (prompt and non-prompt b-hadron cascade production), and is defined as:

$$
\tau=\frac{L_{x y} \cdot m_{J / \psi}^{\mathrm{PDG}}}{\left|p_{\mathrm{T}}\right|}
$$

where $L_{x y}$ is the transverse flight length from the primary vertex defined as the one with highest $\sum p_{\mathrm{T}}$ of tracks and $m_{J / \psi}^{\mathrm{PDG}}$ is the world average invariant mass of the $J / \psi$. The prompt $\chi_{c J}$ candidates have $\tau$ compatible with 0 . A simultaneous $\Delta \mathrm{m}$ - lifetime fit is performed to extract number of candidate events for prompt and non-prompt production, where the mass difference $\Delta \mathrm{m}=\mathrm{m}\left(\mu^{+} \mu^{-} \gamma\right)-\mathrm{m}\left(\mu^{+} \mu^{-}\right)$ is used to discriminate between the $\chi_{c 1}$ and $\chi_{c 2}$ states. The $\Delta \mathrm{m}$ and lifetime distributions for the kinematic region of $10 \leq p_{\mathrm{T}}^{J / \psi}<30 \mathrm{GeV}$ and $\left|y^{J / \psi}\right|<0.75$ are shown in figure 1 . The differential cross sections for the prompt and non-prompt $\chi_{c 1}$ and $\chi_{c 2}$ production in a given bin are calculated as

$$
\frac{d \sigma\left(\chi_{c J}\right)}{d p_{\mathrm{T}}^{\chi_{c J}}} \cdot \mathcal{B}\left(\chi_{c J} \rightarrow J / \psi \gamma\right) \cdot \mathcal{B}\left(J / \psi \rightarrow \mu^{+} \mu^{-}\right)=\frac{N_{J}}{\mathcal{L} \cdot \Delta p_{\mathrm{T}}^{\chi_{c J}}},
$$




\section{EPJ Web of Conferences}

where $\mathcal{L}$ is the integrated sample luminosity and $\Delta p_{\mathrm{T}}^{\chi_{c J}}$ represents the $p_{\mathrm{T}}$ bin width.

To recover the true number of $\chi_{c J}$ s, each reconstructed event is weighted by a factor which includes the detector kinematic acceptance, data-driven measurements of the trigger efficiency and the di-muon reconstruction efficiency. In addition, the photon conversion reconstruction efficiency is estimated using a Monte Carlo simulation. The measured cross section is shown in figure 2 for prompt $\chi_{c}$ and in figure 3 for the non-prompt case. The cross-section results are also measured as a function of $p_{T}^{J / \psi}$.
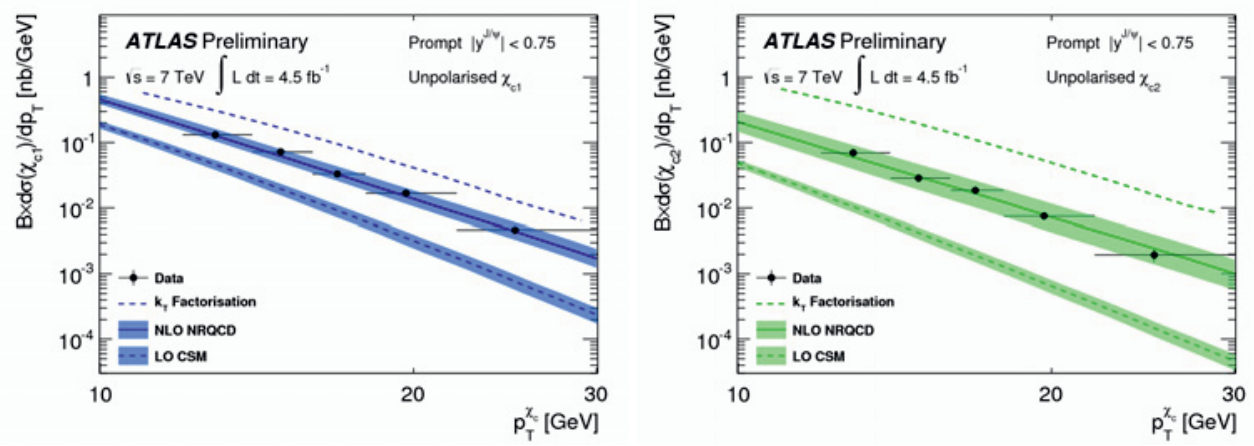

Figure 2. The differential cross sections for prompt $\chi_{c 1}$ (left) and $\chi_{c 2}$ (right) production shown as a function of $p_{T}^{\chi_{c}}$. The predictions of NLO NRQCD model, the $k_{T}$ factorisation model and the LO CSM are compared to the measurements [6]. The factor B in plots denotes the product of branching fractions: $\mathcal{B}\left(\chi_{c J} \rightarrow J / \psi \gamma\right) \cdot \mathcal{B}(J / \psi \rightarrow$ $\mu \mu)$.
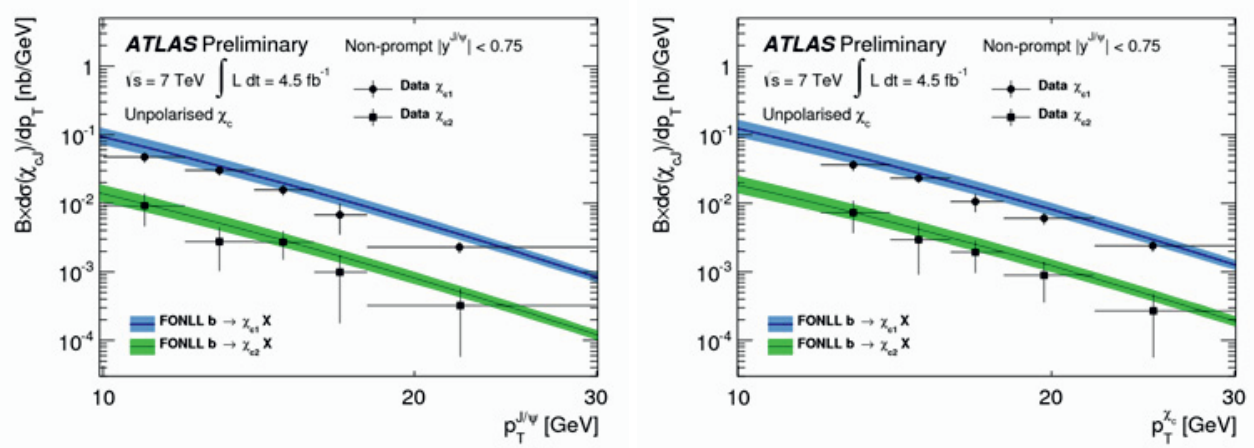

Figure 3. Differential cross sections for the non-prompt $\chi_{c 1}$ (left) and $\chi_{c 2}$ (right) production shown as a function of $p_{T}^{\chi_{c}}$. The measurements are compared to the predictions of the FONLL model [6].

The acceptance (and therefore the measured cross section) depends significantly on the production polarisation of the $\chi_{c J}$ system, which is not currently known for LHC conditions. The corresponding uncertainty is estimated by calculating the weighting factors for maximal polarisation scenarios and re-evaluating the cross section for each one. The zero-polarisation scenario is used for the central values of the cross section, and the variations around this are taken as a theoretical uncertainty. In the following plots, the error bars represent the total uncertainty of the measurement. The results show that in the case of prompt production (figure 2), the next-to-leading Order (NLO) NRQCD is in 
good agreement with the data, while the $k_{\mathrm{T}}$ factorisation model grossly overestimates the measured production cross section, in contrast to the leading order (LO) CSM which predicts significantly lower cross sections, because the higher order corrections are probably large. In the case of non-prompt production, the Fixed Order Next-to-Leading Logarithm (FONLL) prediction is in general agreement with the data, with statistical uncertainties limiting the precision.

\subsection{The fraction of the prompt $J / \psi$ produced in $\chi_{c}$ decays}

The measurements of the prompt $\chi_{c 1,2}$ production cross section are combined with existing measurements of the prompt $J / \psi$ production [7] to estimate the fraction of $J / \psi \mathrm{s}$ which are produced in the $\chi_{c}$ decays. The results are presented in bins of $p_{\mathrm{T}}$. The measurements show that around $20-30 \%$ of $J / \psi$ mesons are produced in the $\chi_{c}$ feed-down. This result is compatible with the LHCb measurements in the overlapping region of the $p_{\mathrm{T}}$ spectra. The results are shown in figure 4 . The NLO NRQCD predictions describe the measurement with a good agreement.

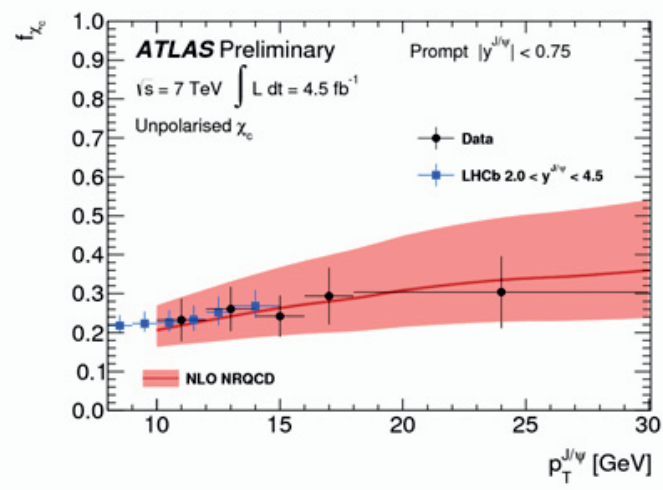

Figure 4. The fraction of prompt $J / \psi$ produced in the $\chi_{c}$ decays as a function of $p_{T}^{J / \psi}$. The measurements are compared to the prediction of NLO NRQCD. The measurement from the LHCb [8] is also shown [6].

\subsection{The $\chi_{c J}$ relative cross sections}

The ratio of the prompt cross sections of $\chi_{c 2}$ and $\chi_{c 1}$ (figure 5, left) are compared against predictions from CS and NLO NRQCD CO calculations. The NLO NRQCD is in general agreement with the data for lower $p_{\mathrm{T}}$ range, while the LO CSM systematically underestimates the measurement. On the other hand, the non-prompt cross section ratio (shown in figure 5, right) is in principle predicted by the relative branching fractions, and it is in good agreement with the CDF data in the $p_{\mathrm{T}}$ spectrum overlap range.

\subsection{The fractions of $\chi_{c 1}$ and $\chi_{c 2}$ produced in the decays of $\mathbf{b}$-hadrons}

The inclusive $\chi_{c}$ production (shown in figure 6) is dominated by the prompt processes unlike the $J / \psi$, where the feed-down from b-hadron decays is substantial. 

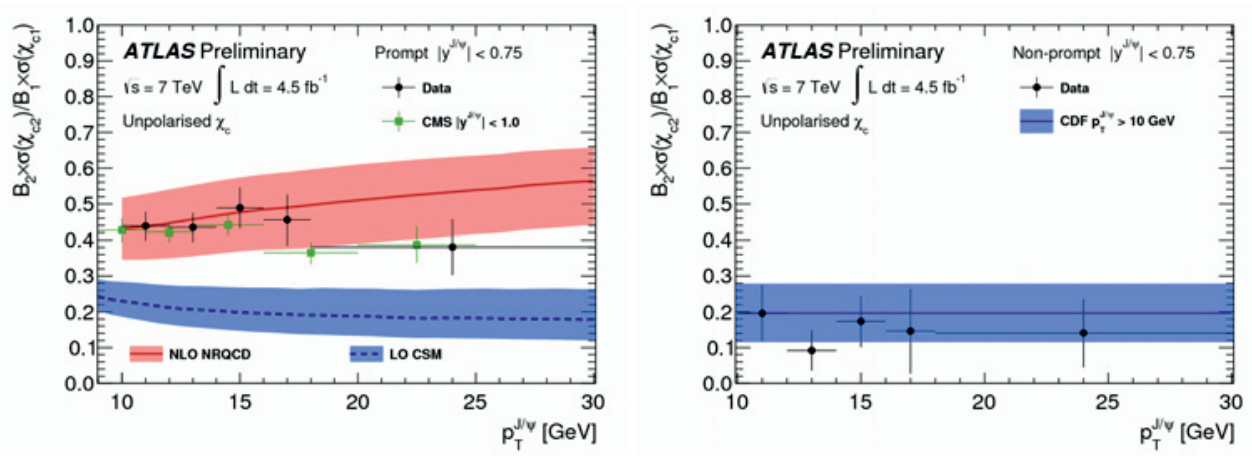

Figure 5. (left)The production cross section of the prompt $\chi_{c 2}$ relative to the prompt $\chi_{c 1}$ measured as a function of $p_{\mathrm{T}}^{J / \psi}$. The measurements are compared to the predictions of NLO NRQCD, LO CSM and to the measurement from CMS [9]. (right) The production cross section of the non-prompt $\chi_{c 2}$ relative to the non-prompt $\chi_{c 1}$ measured as a function of $p_{T}^{J / \psi}$. The measurement from CDF [10] is also shown. The factors $B_{1}$ and $B_{2}$ on both plots represent the branching ratios $B_{J}=\mathcal{B}_{J}\left(\chi_{c J} \rightarrow J / \psi \gamma\right)$ [6].

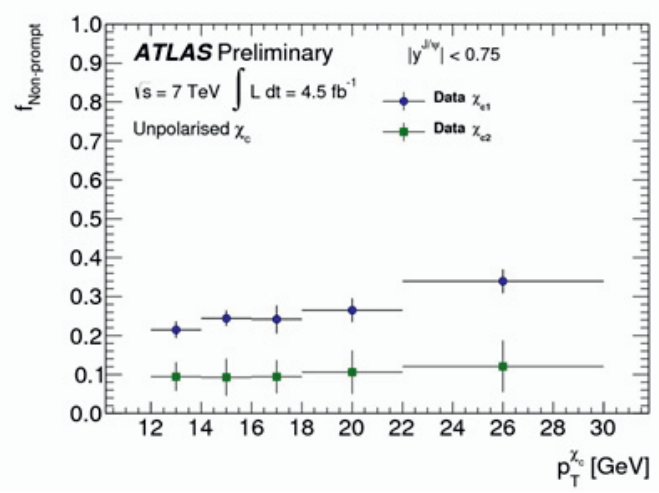

Figure 6. The fractions of $\chi_{c 1}$ and $\chi_{c 2}$ produced in the decays of b-hadrons measured as a function of $p_{T}^{\chi_{c}}$ [6].

\section{Measurement of the $B^{ \pm} \rightarrow \chi_{c 1} K^{ \pm}$branching fraction}

The branching fraction $\mathcal{B}\left(B^{ \pm} \rightarrow \chi_{c 1} K^{ \pm}\right)$is measured using the $B^{ \pm} \rightarrow J / \psi K^{ \pm}$decay as a reference. The final states are identical except for a photon, which allows the direct test of the Monte Carlo-derived photon conversion efficiency corrections, used in the previous measurements presented in this paper. The branching ratio is measured using the relation:

$$
\mathcal{B}\left(B^{ \pm} \rightarrow \chi_{c 1} K^{ \pm}\right)=\mathcal{A} \cdot \frac{N_{\chi_{c 1}}^{B}}{N_{J / \psi}^{B}} \cdot \frac{\mathcal{B}\left(B^{ \pm} \rightarrow J / \psi K^{ \pm}\right)}{\mathcal{B}\left(\chi_{c 1} \rightarrow J / \psi \gamma\right)},
$$

where $\mathcal{A}$ is the factor which corrects for the different detector acceptances of the two decay channels, $N_{\chi_{c 1}}^{B}$ and $N_{J / \psi}^{B}$ are the fitted signal yields for the $\chi_{c 1}$ decay channel and the reference channel. The $\chi_{c 1}$ and $J / \psi$ event selection criteria are kept as close to the inclusive $\chi_{c}$ measurements as possible. Both decays are reconstructed within the kinematic region of $10<p_{\mathrm{T}}^{J / \psi}<30 \mathrm{GeV}$ and $\left|y_{J / \psi}\right|<0.75$. 
The candidate events in both channels are weighted to correct for the trigger efficiency, muon reconstruction efficiency and in the case of the $B^{ \pm} \rightarrow \chi_{c 1} K^{ \pm}$also for the conversion probability and reconstruction efficiency using the same corrections derived for the inclusive $\chi_{c}$ production measurement. The fit results are shown in figure 7.
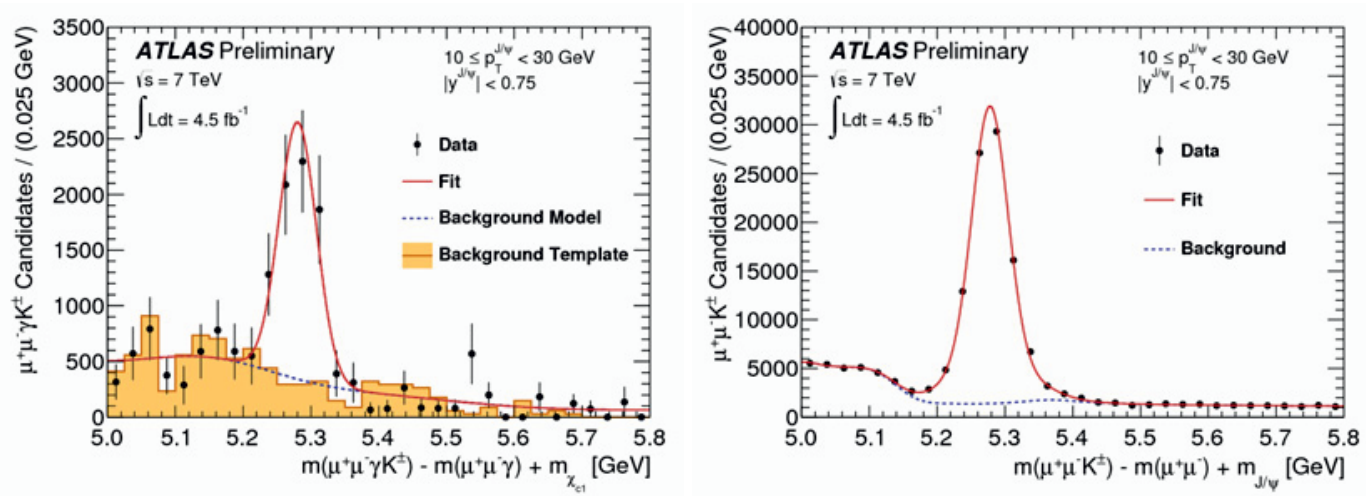

Figure 7. The results of fits to the $m\left(\mu \mu \gamma K^{ \pm}\right)-m(\mu \mu \gamma)+M_{\chi c 1}$ (left) and $m\left(\mu \mu K^{ \pm}\right)-m(\mu \mu)+M_{J / \psi}$ (right) distributions of the selected $B^{ \pm}$decay candidates. The background template derived from MC simulation is shown as the shaded histogram in the left figure [6].

The measured branching fraction is $\left(B^{ \pm} \rightarrow \chi_{c 1} K^{ \pm}\right)=(4.8 \pm 0.6$ (stat. $) \pm 0.6($ syst. $\left.)\right) \times 10^{-4}$. This value is in good agreement with the current world average value of $(4.79 \pm 0.23) \times 10^{-4}$ [3], strongly suggesting that the simulation-derived photon conversion probabilities used in the measurements of the $\chi_{c J}$ production cross sections are valid.

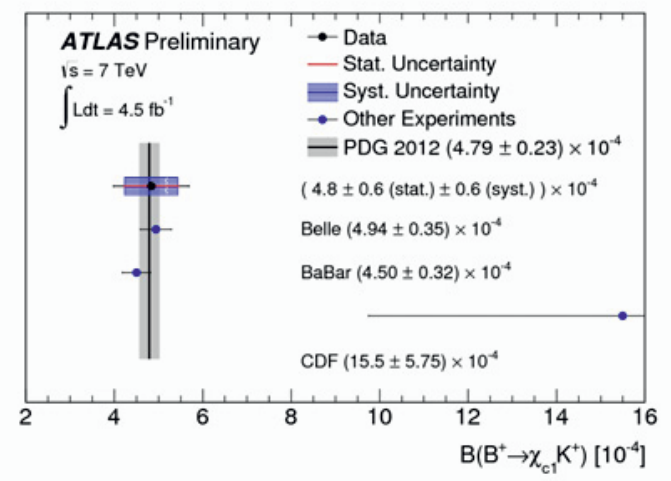

Figure 8. The measurement of the branching fraction $B\left(B^{ \pm} \rightarrow \chi_{c 1} K^{ \pm}\right)$is compared to the measurements of other experiments and to the PDG world average [6]. 


\section{Conclusions}

The cross sections for both prompt and non-prompt $\chi_{c 1}$ and $\chi_{c 2}$ production are measured in $p p$ collisions at the centre-of-mass energy of $7 \mathrm{TeV}$ with the ATLAS detector at the LHC. The production rate of the $\chi_{c 2}$ state relative to the $\chi_{c 1}$ state is measured for both prompt and non-prompt production as a function of $J / \psi$ transverse momentum. The measurements of the prompt $\chi_{c J}$ are combined with existing ATLAS measurements of the prompt $J / \psi$ production to obtain the fraction of the prompt $J / \psi$ produced in the feed-down from $\chi_{c J}$ decays. The fractions of $\chi_{c 1}$ and $\chi_{c 2}$ produced in the decays of b-hadrons are also presented as functions of transverse momentum. The measurements of the prompt $\chi_{c J}$ production are compared to the theoretical predictions of the NLO NRQCD CO model, the $k_{\mathrm{T}}$ factorisation approach and the CS model. The NRQCD CO predictions are found to be in general agreement with the measurements. The $k_{\mathrm{T}}$ factorisation approach predicts larger cross section than the measurement, while the LO CSM prediction significantly underestimates the data. The measurements of the non-prompt $\chi_{c J}$ production are found to be consistent with the FONLL predictions. The branching ratio $\mathcal{B}\left(B^{ \pm} \rightarrow \chi_{c 1} K^{ \pm}\right)=\left(4.8 \pm 0.6(\right.$ stat . $\pm 0.6($ syst. $)) \times 10^{-4}$ is also measured using the same dataset and $\chi_{c J}$ event selection. The measured value is found to be in good agreement with the world average and provides a confirmation of the conversion reconstruction efficiencies derived from the simulation.

\section{Acknowledgements}

We acknowledge the support of the Ministry of Education, Youth and Sports of the Czech Republic under the projects 1P04LA212, LA08032 and LG13009.

\section{References}

[1] N. Brambilla et al., Heavy quarkonium: progress, puzzles, and opportunities, Eur. Phys. J. C71 82-86 (2011), [arXiv:1010.5827v3].

[2] CMS Collaboration, Measurement of the prompt $J / \psi$ and $\psi(2 \mathrm{~S})$ polarizations in pp collisions at $\sqrt{s}=7 \mathrm{TeV}$, Phys. Lett. B727 (2013) 381-402, [arXiv:1307.6070].

[3] Particle Data Group, J. Beringer et al., Review of Particle Physics (RPP), Phys. Rev. D86 (2012) 010001.

[4] Y.-Q. Ma, K. Wang, and K.-T. Chao, QCD radiative corrections to $\chi_{c J}$ production at hadron colliders, Phys. Rev. D83 (2011) 111503.

[5] ATLAS Collaboration, The ATLAS Experiment at the CERN Large Hadron Collider, 2008 JINST 3 S08003.

[6] ATLAS Collaboration, Measurement of $\chi_{c 1}$ and $\chi_{c 2}$ production with $\sqrt{s}=7 \mathrm{TeV}$ pp collisions at ATLAS, ATLAS-CONF-2013-095, http://cdsweb.cern.ch/record/1596292.

[7] ATLAS Collaboration, Measurement of the differential cross-sections of inclusive, prompt and non-prompt $J / \psi$ production in proton-proton collisions at $\sqrt{s}=7 \mathrm{TeV}$, Nucl. Phys. B 850 (2011) 387-344.

[8] LHCb Collaboration,Measurement of the ratio of prompt $\chi_{c}$ to $J / \psi$ production in $p p$ collisions at $\sqrt{s}=7$ TeV, Phys. Lett. B718 (2012) 431-440, [arXiv:1204.1462].

[9] CMS Collaboration, Measurement of the relative prompt production rate of $\chi_{c 2}$ and $\chi_{c 1}$ in $p p$ collisions at $\sqrt{s}=7 \mathrm{TeV}$, Eur. Phys.J. C72 (2012) 2251, [arXiv:1210.0875].

[10] CDF Collaboration, Measurement of $\sigma\left(\chi_{c 2}\right) \mathcal{B}\left(\chi_{c 2} \rightarrow J / \psi \gamma\right) / \sigma\left(\chi_{c 1}\right) \mathcal{B}\left(\chi_{c 1} \rightarrow J / \psi \gamma\right)$ in $p \bar{p}$ collisions at $\sqrt{s}=1.96 \mathrm{TeV}$, Phys. Rev. Lett. 98 (2007) 232001, [hep-ex/0703028]. 